\title{
Cancer Metabolomics in the Context of Systems Biology
}

\section{Ariel Fernández $z^{1,2,3}$}

${ }^{1}$ Argentine Mathematics Institute, CONICET (National Research Council), Buenos Aires 1083, Argentina

${ }^{2}$ Collegium Basilea, Institute for Advanced Study, CH 4053 Basel, Switzerland

${ }^{3}$ Ariel Fernández Consultancy, Avenida del Libertador 1092, Buenos Aires 1112, Argentina

Keywords: Cancer; Genomics; Transcriptomics; Proteomics; Interactomics; Metabolomics; Networks; Profiling; Biomarker; Fingerprinting; Personalized medicine; Integrative biology

\section{Editorial}

In spite of significant forays for gathering information at the "omics" level during the current postgenomics era, an effective integration and processing of this information to improve human medicine is still felt to be lacking. On the other hand, metabolomics aims at generating a direct chemical fingerprint of all the cellular processes taking place in a living organism. As such, it strives to provide a systematic profile of biochemical intermediates and products, and hence integrates and distills information from the genomic, transcriptomic, proteomic, fluxomic and interactomic levels.

Because metabolomics makes strides through such natural integration and distillation of omics information, it lends itself naturally to improve medicine in a manner in which systems biology approaches can prove particularly helpful. In spite of commendable preliminary efforts in this direction, a significant gap still exists between highthroughput "omics" acquisition of information and its systems-level interpretation [1].

While profiling and fingerprinting platforms furnish large-scale omics data, systems biology should in principle enable network building and dynamic integration of the information. Thus, genomic, transcriptomic and proteomic large-scale data represent valuable input data, while metabolic intermediate levels, being direct markers of cellular activity, serve as the ultimate testing ground for the systems biology models. This level of model building may ultimately lead to the identification of master regulators [2]. Yet, at a more general level, it is at this point unclear what level of omics information coarsegraining will become necessary and sufficient to enable integration and correlation of data in a manner that will enrich and empower the metabolomics output [3].

It is likely that a synergic relation between metabolomics and systems biology may ultimately trigger a revolution in personalized medicine. The diagnostic use of metabolic multiplexing readouts will become significantly more efficacious when integrated with the patient omics profile enabled or facilitated by system biology models. The synergy between metabolomics and systems biology will fundamentally transform diagnostics, disease prevention, and therapy.
Thus, visionary thinking in the field of metabolomics invites integration with functional genomics, transcriptomic and proteomics platforms enabled by network modeling. In this regard, this author genuinely concurs with the current "mantra" that technological innovation is likely to lead the way to scientific discovery. In line with this thinking, our journal Metabolomics: Open Access is expected to become a suitable venue to report novel profiling platforms and fingerprinting technologies, but also a forum that welcomes contributions that combine experimental and computational approaches, as inter-discipline cross-fertilization is the only viable way to achieve a complete understanding of cellular processes.

These efforts may prove germane to understand cancer at the molecular level. This area has been traditionally focused on transformations in signaling molecules that turn homeostatic cells into malignant ones. However, it is becoming more apparent that dynamic relational analysis of multi-dimensional omics data will be needed to gain an effective understanding of the molecular etiology of the disease. As indicated above, metabolomics remains naturally at the heart of this integration, but since it only captures end products and substrates of cellular chemical processes, the metabolomics data needs to be fleshed out at the different omics levels to fully understand its origin and implications. Much of this integration effort will be hopefully directed at (1) Assess the effect of genomic and transcriptomic aberrations on dysfunctional cell signaling architectures and (2) Investigate network aberrant re-wiring, mapping the changes of the individual proteins responsible for spatiotemporal aberrant re-design in order to better understand cancer biology at the systems level.

\section{References}

1. Palsson B, Zengler $K$ (2010) The challenges of integrating multi-omic data sets. See comment in PubMed Commons below Nat Chem Biol 6: 787-789.

2. Filipp FV (2013) Cancer metabolism meets systems biology: Pyruvate kinase isoform PKM2 is a metabolic master regulator. See comment in PubMed Commons below J Carcinog 12: 14.

3. James LP (2013) Metabolomics: integration of a new "omics" with clinical pharmacology. See comment in PubMed Commons below Clin Pharmacol Ther 94: 547-551.
*Corresponding author: Ariel Fernández, Argentine Mathematics Institute, CONICET (National Research Council), Buenos Aires 1083, Argentina, Tel: 818 964 6624; E-mail: ariel@afinnovation.com

Received July 9, 2013; Accepted July 11, 2014; Published July 18, 2014

Citation: Fernández A (2014) Cancer Metabolomics in the Context of Systems Biology. Metabolomics 4: e127. doi:10.4172/2153-0769.1000e127

Copyright: (c) 2014 Fernández A. This is an open-access article distributed under the terms of the Creative Commons Attribution License, which permits unrestricted use, distribution, and reproduction in any medium, provided the original author and source are credited. 